\title{
A viscoelastic system for determining acoustical and mechanical parameters of the bone
}

\author{
Haydar Aygün \\ School of the Built Environment and Architecture, London South Bank University, 103 \\ Borough Road, London, UK. Email: aygunh@lsbu.ac.uk or haydar-aygun@hotmail.com
}

\begin{abstract}
Human bones are energy absorbing complex composite structures that have an irregular hollow structure filled with marrow and surrounded by soft tissue and muscles. To date, no satisfactory evidence exists either supporting or refuting the usefulness of vibro-acoustic technique to determine the acoustical and mechanical parameters of the bone. The aim of the paper is to use acoustic wave propagation techniques and viscoelastic bone system to analysis and assess the acoustical and mechanical properties of human bone, which may be used to monitor the bone condition and diagnose the osteoporosis at an early stage, by demonstrating if variations in sound propagation through the bone can be detected.
\end{abstract}

\section{Introduction}

Human bones are energy absorbing complex composite structures that have an irregular hollow structure filled with marrow and surrounded by soft tissue and muscles. They have two types of structure, cortical and cancellous bone, both having the same mineralized collagen composition. Cortical bone may generally be considered solid; cancellous bone consists of a complex open-celled porous network of rod- and plate-shaped elements termed trabeculae. The porosity of human cancellous bone ranges between $70 \%$ and $95 \%$, the remaining volume being perfused with bone marrow ${ }^{1}$. Bone quality is a composite of properties that make bone resist fracture, such as its microarchitecture, accumulated microscopic damage, the quality of collagen, mineral crystal size, and bone turnover ${ }^{2}$.

During childhood, more bone is added than taken away. Whilst early adulthood, the amounts removed and added are the same. If however, more bone is removed than added, we have a condition called osteoporosis. It literally means 'porous bone' and describes a period of largely asymptomatic bone loss leading to skeletal fragility and increased risk of fracture. It is caused by hormonal imbalance (oestrogen \& testosterone) and long-term cortico-steroid use. It is also caused by low bone mass, as well as a weakened structure. Osteoporosis has been recognized as an established and well-defined disease that affects more than 75 million people in the Europe, United States, and Japan ${ }^{3}$. Osteoporosis causes more than 8.9 million fractures annually worldwide, of which more than 4.5 million occur in the Europe and United States. Osteoporosis is not only a major cause of fractures, it also ranks high among diseases that cause people to become bedridden with serious complications. These complications may be life threatening in ageing people because it is estimated that the burden of osteoporosis will increase four-fold by the year $2050^{4}$. The prevention of osteoporosis and its associated fractures is a requirement to maintain the independence, well-being, and quality of life of the elderly population in World.

Dual energy X-ray absorptiometry (DXA) provides the bone mineral density (BMD), and is used to diagnose osteoporosis ${ }^{5,6}$. However, DXA predicts the fracture risk ${ }^{7,8}$. DXA is a non- 
volumetric measure of bone quantity but it is not a non-volumetric measure of bone quality ${ }^{5}$. X-ray quantitative computed tomography (QCT) allows volumetric bone density assessment 9,10 . But it is an expensive technique, and it delivers a significantly higher radiation dose ${ }^{11}$. For in-vitro bone samples, micro-computed tomography $(\mu \mathrm{CT})$ is considered the gold standard for bone microstructure imaging ${ }^{12}$. Langton et al. ${ }^{13}$ have described the clinical utility of Quantitative Ultrasound (QUS) to assess the mechanical of bone. QUS parameters of velocity and attenuation are dependent upon bone quantity and bone quality, providing a prediction of fracture risk comparable to DXA ${ }^{14-15}$. Previous works have investigated the mechanical properties of bone using vibration ${ }^{16-18}$ and ultrasound ${ }^{19}$ to assess bone fragility on bone.

The inherent anisotropy of cancellous bone means that the acoustical properties vary with transmission direction. Tortuosity deduced from audio-frequency measurements in air-filled bone replicas, assuming rigid-porous behaviour, has shown a strong anisotropy ${ }^{20}$. To predict fast wave transmission, it is also necessary to allow for elastic anisotropy. A method of including the effects of anisotropy in Biot model ${ }^{21-23}$ introduces an angle dependent tortuosity. Biot theory has been used extensively to describe the wave propagation in cancellous bone ${ }^{24-}$ 32. It was specifically developed to describe acoustic wave propagation in fluid-saturated porous elastic media ${ }^{33-34}$. Biot theory predicts two compressional waves (fast and slow waves), when the waves propagating through the solid frame of bone and marrow are in-phase and outof-phase respectively, and a shear wave. It allows for an arbitrary microstructure, with separate motions considered for the solid elastic framework (bone) and the interspersed fluid (marrow), induced by the ultrasonic wave, and also includes energy loss due to viscous friction between solid (bone) and fluid (marrow).

The common technique used to determine the mechanical properties of the bone is conventional mechanical testing which is invasive and destructive. The strength of the bone can be estimated from measured stiffness of the bone using mechanical test although it is inappropriate in-vivo. The mechanical behaviour of the bone structure ${ }^{35}$ has been predicted using finite element analysis (FEA) which is a non-destructive computer software. The bone imaging techniques in vivo has combined with FEA ${ }^{36-38}$. Most recently Langton et al. ${ }^{39}$ has combined ultrasound computed tomography (UCT) with FEA to predict the stiffness of bone. They have demonstrated that UCT_FEA based upon quantitative attenuation images provided a comparable estimation of gold standard mechanical test stiffness of $84 \%$ compared to microCT-FEA. Current recommendations for the assessment of patients for bone mineral density (BMD) and fracture risk have several difficulties, and they are not suitable for international use. To date, no satisfactory evidence exists either supporting or refuting the usefulness of the vibro-acoustic technique.

The aim of this paper is to use the vibro-acoustic technique to analyse and assess the acoustical and mechanical properties of human bone by demonstrating if variations in sound propagation through the bone can be detected. Firstly, a viscoelastic bone system is modelled to estimate mechanical properties of the bone. Secondly, the force generated by an impact hammer is applied to the human tibia in vivo and its corresponding response is detected by using an accelerometer at $15 \mathrm{~cm}$ distance from the impact hammer. The distance between the accelerometer and the impact hammer is increased and same measurements are repeated to see if the structural borne sound waves are attenuated while they are propagating through the human tibia. A male human tibia is used for vibro-acoustics measurements, and results from bone tibia are presented in this paper to explain the process of determining acoustical and mechanical parameters of human tibia. Thirdly, measurements are performed on sawbones with and without perforations to determine the resonance frequencies from transfer function curve and to see if fundamental, second and third resonance frequencies, mentioned in previous works carried out on dry human bone and dog femora ${ }^{17-18}$, can be observed in their frequency 
dependent function. Finally, modal analysis of the human bone and replica bone (sawbone) are carried out using the viscoelastic bone system to estimate mechanical properties of the bone from acoustical parameters deduced from frequency dependent transfer function.

\section{Theory of the bone vibration}

Almost all-natural materials are viscoelastic. They have both damping and strain energy storage capabilities. Human bones can be considered as viscoelastic complex composite structures because they have both damping and energy storage capabilities. A human bone system (human tibia) that consists of bone, soft tissue, marrow and muscles has one peak amplitude and one resonance frequency ${ }^{40}$. Therefore, this system can be modelled as a one-degree-of-freedom system.

If a force is applied to a viscoelasticly damped mass-spring system (human bone) as shown in Figure 1, the equation of motion can be obtained using Newton's second law. The equation of motion of a single degree-of-freedom system with viscoelastic damping under a sinusoidal excitation $F(t)=F_{1} \cos (2 \pi f t)$, is expressed ${ }^{41}$ as

$$
m \ddot{x}+\bar{k} x=F_{1} \cos (2 \pi f t)
$$

where $m$ is the total mass of the human bone (tibia), $F_{1}$ is the amplitude of the sinusoidal excitation force, $f$ is the force frequency, $t$ is the time, $\bar{k}$ is the complex stiffness of the bone system, and given by $\bar{k}=k(1+i \eta)$, where $k$ is the normal stiffness of the bone and it is given by the total mass of the bone system multiplied by square of natural frequency of the bone system, and $\eta$ is the loss factor (or loss coefficient) of the bone. The real part of complex stiffness indicates how much strain energy can be stored by the bone system, and the imaginary part of it shows the energy dissipation capability of the system. The total mass of the bone system is given by

$$
m=m_{t}+m_{m}+m_{b}^{\prime}+m_{m w}^{\prime}
$$

where $m_{t}$ is the mass of the soft tissue surrounding the bone, $m_{m}$ is the mass of the muscles, $m_{b}{ }_{b}$ is the porosity dependent mass of the bone and it is given by $m^{\prime}{ }_{b}=m_{b}(1-\emptyset)$, where $m_{b}$ is the mass of the bone itself, and $\varnothing$ is the porosity of the bone, $m^{\prime}{ }_{m w}$ is the porosity dependent mass of the marrow in the bone and it is given by $m_{m w}^{\prime}=m_{m w}(1+\emptyset)$, where $m_{m w}$ is the mass of the marrow.

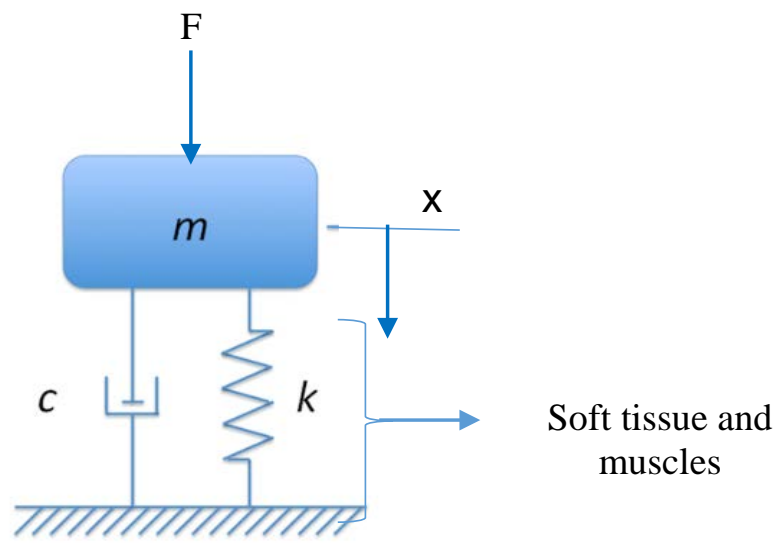

Figure 1: Viscoelasticly damped human bone system. 
The steady-state response ${ }^{41}$ of the bone vibration system for equation 1 is given by the particular solution, which is expected to be harmonic:

$$
x=X \cos (2 \pi f t-\phi)
$$

where $X$ is the amplitude of the forced vibration of the bone, and $\phi$ is the phase angle of the steady-state response. Equation 3 represents simple harmonic motion of the bone system at force frequency with a displacement vector which lags the force vector by $\phi$ that is the motion occurring after the force is applied to the bone.

The response of the damped single degree of freedom of the bone system excited by a harmonic force ${ }^{41}$ depends on the static deflection, damping ratio of the system and frequency ratio of the system. The response of the system is given as follow:

$$
X=\frac{F_{1} / \bar{k}}{\sqrt{\left(1-r_{f}^{2}\right)^{2}+\left(2 \xi r_{f}\right)^{2}}}
$$

Where $r_{f}$ is the frequency ratio given by $\frac{f}{f_{n}}, F_{1} / \bar{k}$ is the static deflection which would be produced by the force $F(t)$, and $f_{n}$ is the undamped natural frequency of the system.

The relationship between driving frequency, undamped natural frequency and damping ratio is given as $f=f_{n}\left(1-2 \xi^{2}\right)^{1 / 2}$, where $\xi$ is the damping ratio of the bone system. The relationship between undamped and damped natural frequencies and damping ratio is given as $f_{d}=$ $f_{n}\left(1-\xi^{2}\right)^{1 / 2}$, where $f_{d}$ is the damped natural frequency of the bone.

\section{Measurements}

Measurements were carried out on the tibia bone which is the second longest bone in the human body to the femur. The tibia is as such composed of a diaphysis and two epiphyses that are the two rounded extremities of the bone. The tibia is ossified from a primary centre for the shaft and a secondary centre for each extremity. The diaphysis is the midsection of the tibia known as the shaft which is triangular in cross section. The epiphyses are the two rounded extremities of the bone known as upper extremity and lower extremity ${ }^{[42]}$.

The human subject who volunteered for experimental study was a healthy 42 years old male person. His weight and height were $79 \mathrm{~kg}$ and $182 \mathrm{~cm}$ respectively. Experimental set-up for vibracoustic bone measurements is given in Figure 2. An impact hammer (PCB086C03) was used to generate a force to vibrate the bone. The responses were detected along the bone surface using an accelerometer (MME-KS901-100). The Impact hammer and accelerometer were connected to signal conditioning units (DJB-VB/01) which were fed to a data acquisition system (NI-USB-4431) which was connected to a computer to save and analyse the data obtained from measurements.

Electronic interference was removed by 10000 acquisition averages per second. Unwanted noise that recorded with applied force and detected responses were filtered by using the Wavelet toolbox in MATLAB. Signals were decomposed to eliminate the noise. The signals were reconstructed for further analysis after the noise was removed from the signals. 


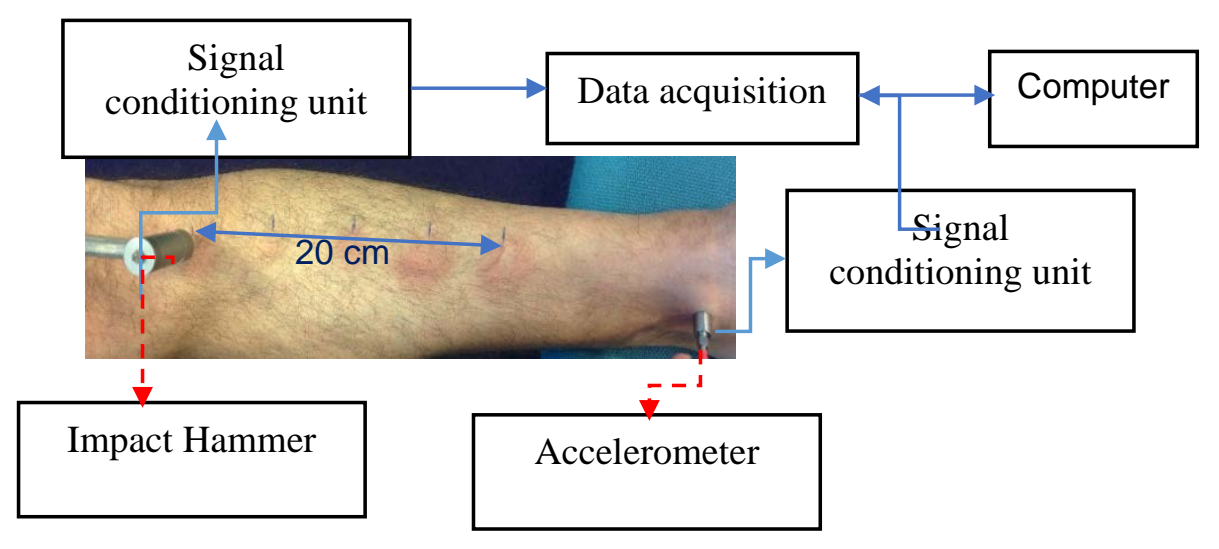

Figure 2: Vibro-acoustics bone measurement set-up.

The force generated by impact hammer was applied to human tibia in vivo, and its responding signals were detected at different positions along surface of the bone using an accelerometer. The responding signals were detected at $15 \mathrm{~cm}, 20 \mathrm{~cm}$ and at the ankle. Vibration of the bone generates structural borne acoustic waves that travel through the bone structure and along the soft tissue covering the bone. The input forces applied to bone are given in Figure 3a, and their corresponding responses are shown in Figure 3b. The amplitude of the responses detected for male tibia is shown in Figure 4. There is slight reduction in the amplitude of the responses throughout the frequency range. Detected responses can't be compared because initial forces applied to bone by an impact hammer are not identical and they are not repeatable.
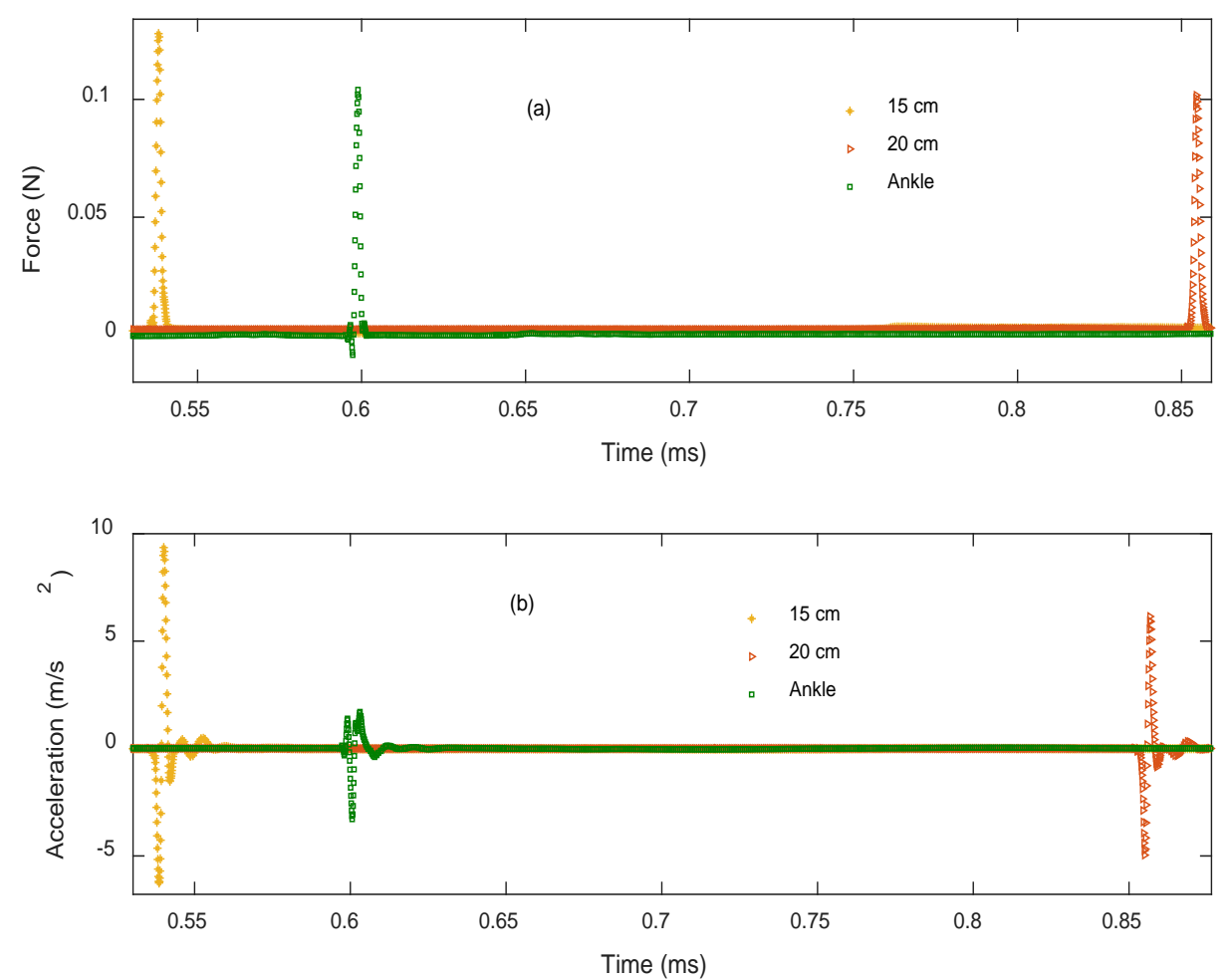

Figure 3: (a) Input force applied to human tibia by an impact hammer, (b) Responses detected by an accelerometer. 


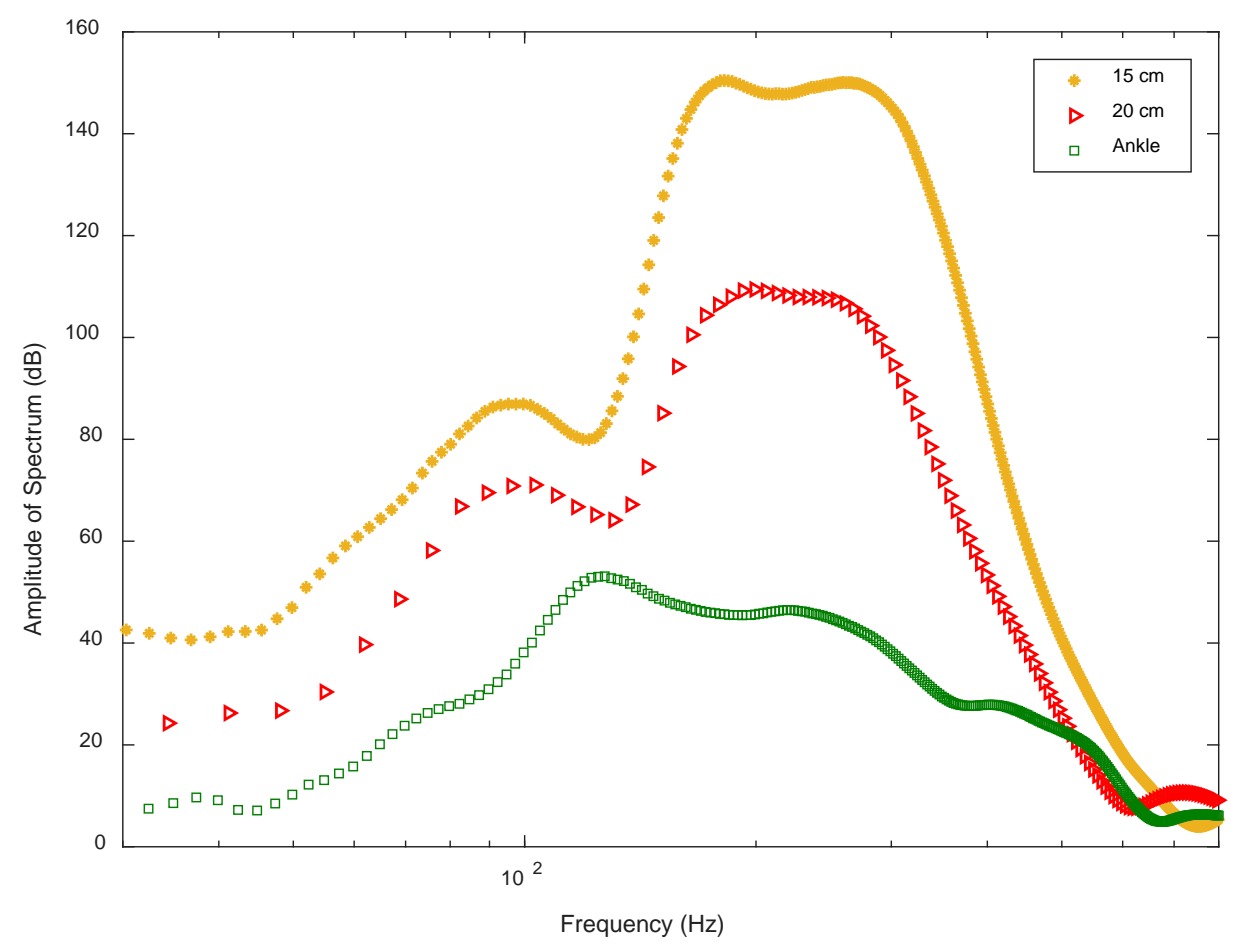

Figure 4: Amplitude of responses detected by an accelerometer.

A transfer function method based on Laplace transforms is used to analyse detected the bone responses in details and to extract acoustical and structural parameters of the bone. The transfer-function approach may be used for solving forced-vibration problems. It relates a system's output to its input and assumes zero initial conditions. Detected signals in the time domain were denoised before they were converted into the frequency domain using a Fast Fourier Transform (FFT). Frequency dependent transfer function of the system is determined using the response of the system and the force applied to the system using the equation below;

$$
H(f)=\frac{A(f)}{F(f)}
$$

where $A(f)$ is the response of the system in the frequency domain, and $F(f)$ is the force applied to system in the frequency domain.

Frequency domain transmission function curves of male tibia are given in Figure 5 . When the distance between the accelerometer and the impact hammer is increased, the amount of structural borne acoustic energy transmitted through the bone mostly reduces throughout the frequency range, and it causes the natural frequency of male bone to shift from higher frequency to a lower frequency.

The soft-tissue surrounding the bone tibia attenuates vibrational force, absorbs sound energy, and behaves as a wave guide allowing the vibration borne sound waves to propagate through it. The attenuation of acoustic energy travelling through bone may be due to the distance between input and outputs, marrow in the bone, muscles, soft tissue surrounding it, and changes in bone diameter along the tibia surface. As it can be seen from Figure 5, only the fundamental resonance frequency of the bending vibration was determined from the measured transfer function curve of the human tibia. The second and third resonance frequencies, mentioned in previous works carried out on dry human bone and dog femora ${ }^{17-18}$, were not observed on human bone despite repeating the same measurements several times. Dry bones behave like a 
porous rigid beam and allows the most of structural borne vibrational energy to be transmitted through its structure. This is most likely due to the lack of any marrow, soft tissue and muscles to absorb sound waves and dampen the structural borne vibration.

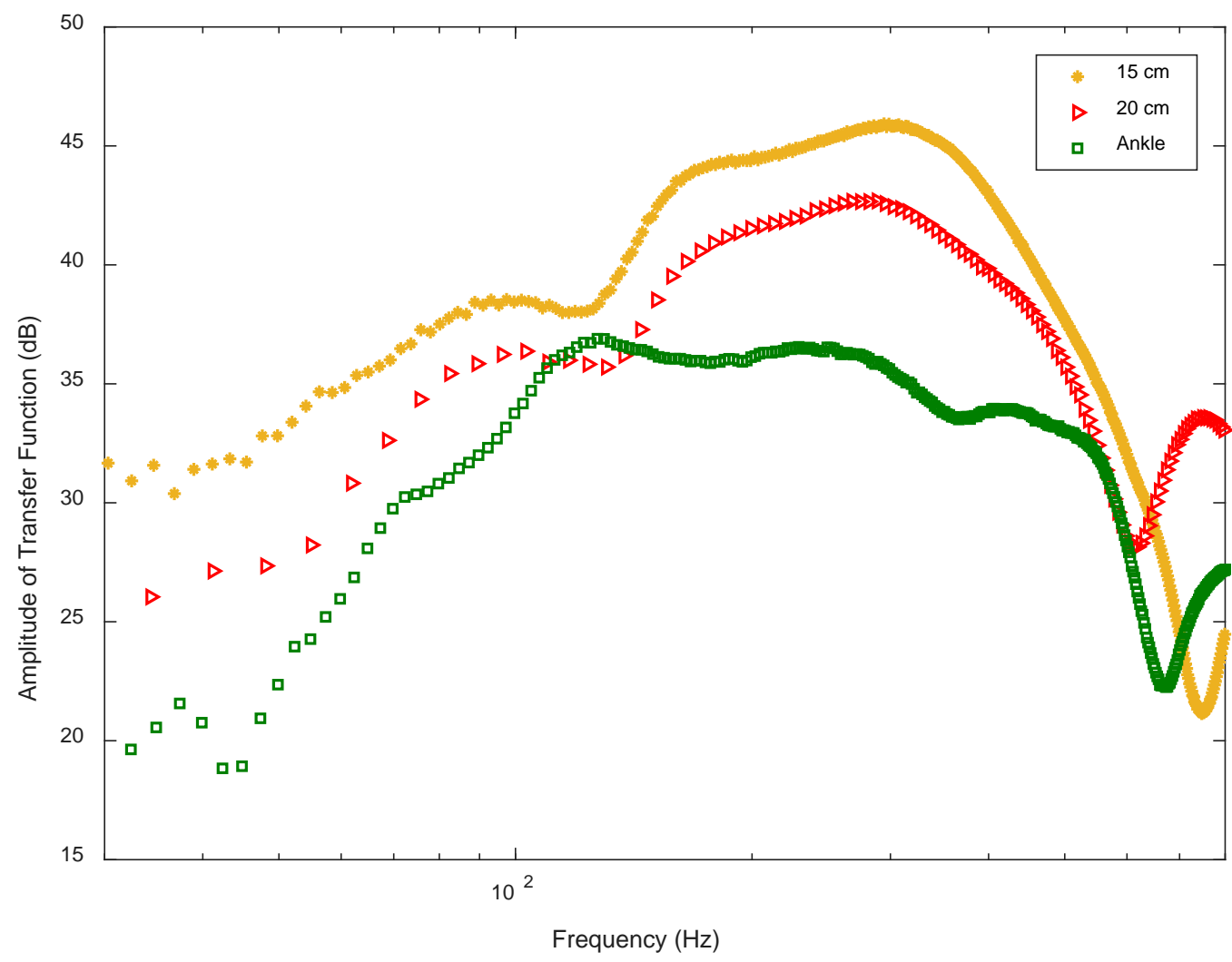

Figure 5: Transfer functions versus frequency for human tibia.

Measurements were carried out on sawbones with and without perforations. The aim of these measurements on sawbones was to determine the resonance frequencies from transfer function curve and to see if second and third resonance frequencies, mentioned in previous works carried out on dry human bone and dog femora ${ }^{17-18}$, were observed in their transfer function response. Sawbone (replica bone) made of solid rigid polyurethane foam does not replicate the structure of human bone. But its materials properties are in the range of human cancellous bone. The density of sawbone is $1.64 \mathrm{~g} / \mathrm{cc}$, with a strength of $106 \mathrm{MPa}$, and a length of $40.5 \mathrm{~cm}$. Initial measurements were carried out on the sawbone without perforating it. Indeed, fundamental, second and third resonance frequencies were observed from the frequency dependent function given by dashed line in Figure 6. Measurements were repeated on the perforated sample, initially with 20 holes of $2 \mathrm{~mm}$ diameter, and then with 40 holes of $2 \mathrm{~mm}$ diameter. The distance between impact hammer and accelerometer was $20 \mathrm{~cm}$. Increasing the perforation ratio of sawbones slightly amplified the amplitude of transfer function at mid-range frequencies while shifting the resonance frequencies to lower values. 


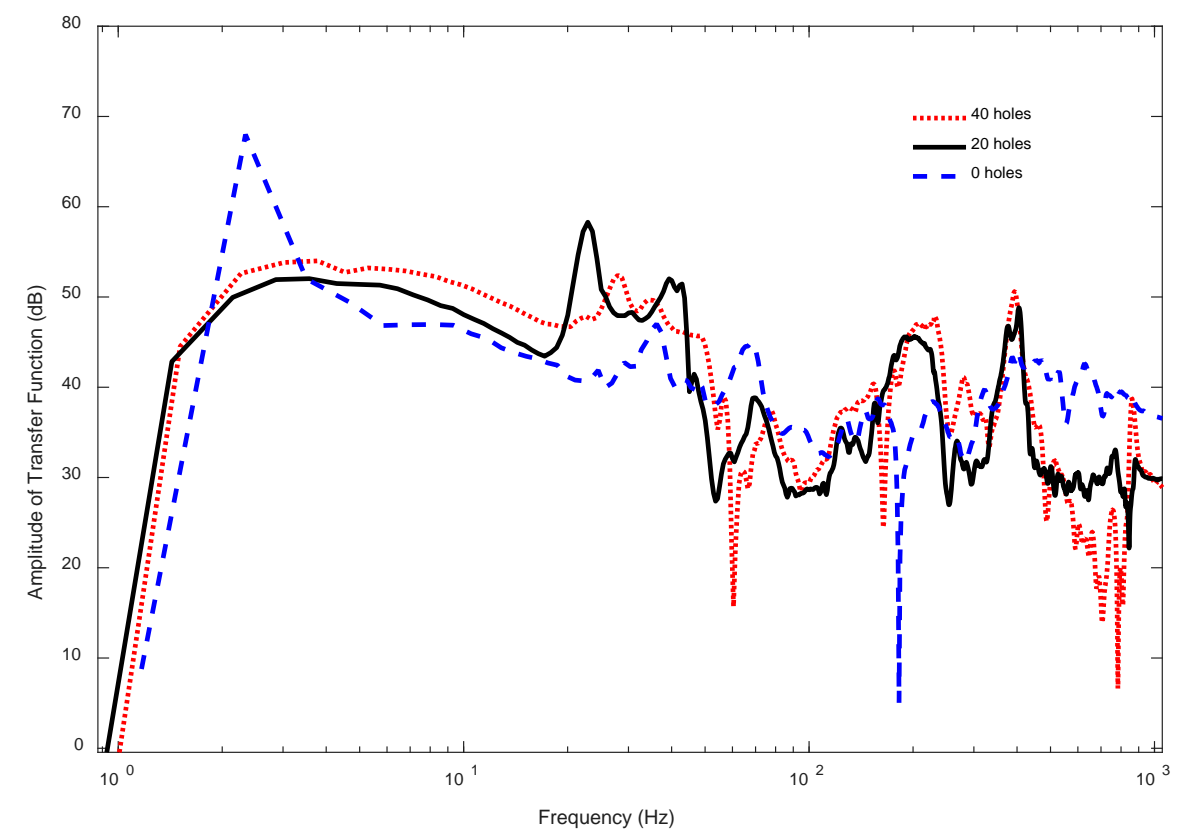

Figure 6: Frequency dependent function of replica bones (sawbones) with and without perforations.

\section{Modal analysis}

\section{A. Damping coefficient}

The transfer function method was used to determine the damped natural frequencies, damping ratios, and mode shapes corresponding to all resonant peaks observed in Figures 5. A MATLAB syntax was used to find peak amplitude of the transfer function. The damping ratio ${ }^{41}$ was found using the equation below;

$$
\xi=\frac{f_{2}-f_{1}}{2 f_{n}}
$$

where the points $f_{1}$ and $f_{2}$, where the amplification factor falls to $\frac{\left|H\left(i f_{n}\right)\right|}{\sqrt{2}}$, are called half-power points because the power absorbed by the damper (soft tissue, muscles, and bone), responding harmonically at a given frequency, is proportional to the square of the amplitude.

The difference between the frequencies associated with the half-power points is called the bandwidth of the system, and satisfies the relation ${ }^{41}$,

$$
\left|H\left(i f_{1}\right)\right|=\left|H\left(i f_{2}\right)\right|=\frac{\left|H\left(i f_{n}\right)\right|}{\sqrt{2}}
$$

The damping ratio corresponding to peak amplitude of transfer function in Figure 7 with resonant frequency $f_{n}$ denotes the modal damping ratio $\xi$. The amplitude of transfer function for the response detected at $20 \mathrm{~cm}$ is $42.69 \mathrm{~dB}$ at resonant frequency, $f_{n}=288.5 \mathrm{~Hz}$. The power absorbed by the visoelastic system at half-power points is $3 \mathrm{~dB}$ less than the peak amplitude of the system at resoance frequency as shown in Figure 7. 


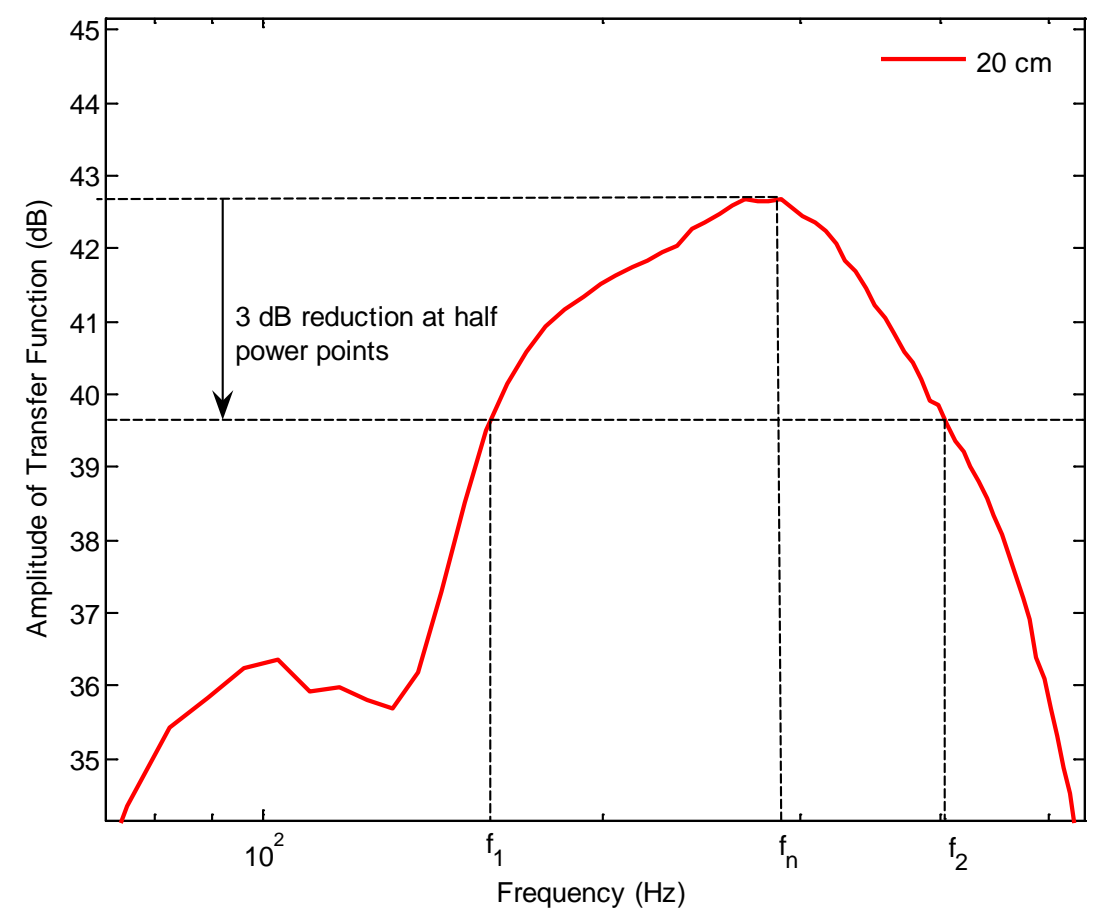

Figure 7: The amplitude of tranfer function response of the tibia bone detected at $20 \mathrm{~cm}$.

\section{B. Loss factor}

The loss factor (or loss coefficient) was originally developed as a measure of intrinsic damping of viscoelastic structures. It is the energy loss per radian to the energy associated to the vibration. The loss factor of the bone system ${ }^{42}$ is defined with respect to steady-state oscillation as follow;

$$
\eta=\frac{E / 2 \pi}{W}
$$

where $E$ is the energy dissipated per cycle of a vibration at frequency $f_{n}$ and equals to $\pi \omega_{n} c X^{2}$ where $c$ is the damping coefficient of the system, $\omega_{n}$ is the undamped radian natural frequency of the system given by $2 \pi f_{n}$, and $W$ is the total energy of the system and it equals to $\frac{1}{2} m \omega_{n}^{2} X^{2}$. The loss factor is related to damping ratio ${ }^{43}$ as;

$$
\eta=\frac{\pi \omega_{n} c X^{2} / 2 \pi}{\frac{1}{2} m \omega_{n}^{2} X^{2}}=2 \xi
$$

The damping capacity $\psi$ is the ratio of the energy dissipated per cycle to the energy present in the system, and given ${ }^{43}$ by;

$$
\psi=\frac{E}{W}=4 \pi \xi
$$

\section{Elasticity of the bone}

If we consider the human tibia used for vibro-acoustic measurements as a simply supported beam of circular cross section $8 \mathrm{~cm}$ and length $45 \mathrm{~cm}$, and assuming the bone tibia tested had a mass of $5 \mathrm{~kg}$, then its elasticity can be estimated using the equation below;

$$
E=\bar{k} \frac{l^{3}}{192 I}
$$


where $\bar{k}$ is given by $m \omega_{n}^{2}(1+i \eta), l$ is the length of human tibia, and $I$ is the area moment of inertia of human bone and it is given by $\pi d^{4} / 64$ where $d$ is the diameter of the bone.

Elasticity of bone tibia can be estimated from natural frequency measurements using equation 11.

\section{Results}

The parameters of human tibia and replica bone (sawbone) were determined using frequency response signals. Increasing the distance between the impact hammer and accelerometer caused slight changes in the bone parameters as shown in Table 1. More acoustic energy was absorbed by the bone system when the structural borne sound waves propagated though the bone. The resonance frequency of the bone was shifted to lower frequency. Damping capacity of the system was reduced because of the changes in damping coefficient and loss factor. The resonance frequency determined from transfer function at $15 \mathrm{~cm}$ and $20 \mathrm{~cm}$ distances from the accelerometer were found to be equal to $294.44 \mathrm{~Hz}$ and $288.5 \mathrm{~Hz}$ respectively. Frequencies $\left(f_{1}\right.$ and $f_{2}$ ) at half-power points are $155.37 \mathrm{~Hz}$ and $401.33 \mathrm{~Hz}$ for response at $15 \mathrm{~cm}$, and 159.82 $\mathrm{Hz}$ and $402.62 \mathrm{~Hz}$ for response at $20 \mathrm{~cm}$ respectively. The reason for these changes in bone parameters could be attributed to the presence of soft tissue attenuating and damping sound waves propagating along the surface of bone tibia. The porous structure of the bone filled with marrow can be another reason for the attenuation of acoustic waves travelling through the bone. This reveals anisotropy in elasticity in human tibia.

Table 1: Bone tibia parameters deduced from transfer function method.

\begin{tabular}{llll}
\hline Parameters & \multicolumn{2}{l}{ Human male bone } & Replica bone \\
\hline & $\mathbf{1 5} \mathbf{~ c m}$ & $\mathbf{2 0 ~} \mathbf{~ m}$ & $\mathbf{2 0} \mathbf{~ c m}$ \\
\hline The peak response & $45.92 \mathrm{~dB}$ & $42.69 \mathrm{~dB}$ & $68 \mathrm{~dB}$ \\
Resonance frequency $\left(f_{n}\right)$ & $294.44 \mathrm{~Hz}$ & $288.5 \mathrm{~Hz}$ & $2.34 \mathrm{~Hz}$ \\
Damping coefficient $(\xi)$ & 0.418 & 0.421 & 0.37 \\
Loss factor $(\eta)$ & 0.836 & 0.842 & 0.74 \\
Damping capacity $(\psi)$ & 5.25 & 5.3 & 4.65 \\
Elasticity $(E)$ & $(3.8+$ i 7) GPa & $(8.6+$ i 15.4) GPa & $(75.6+$ i 11.1) MPa
\end{tabular}

\section{Conclusion and further work}

An investigation was carried out on human bone in-vivo to detect the variation of sound propagation in the bone using structural borne acoustic wave technique. The tibia of a male human subject was used for vibro-acoustics measurements to determine acoustical and mechanical parameters of it. The acoustics wave technique was used to deduce acoustical properties of bone from frequency depend transfer function of the system while a viscoelastic bone system is modelled and used to estimate mechanical properties of the bone. Varying the 
distance between the accelerometer and impact hammer mostly changes the natural frequency of human tibia, especially at lower frequencies. The resonances (second and third resonance frequencies) observed in measurements undertaken on dry bones and sawbones were not seen in frequency dependent transfer function of human bone surrounded with soft tissue and muscles except for fundamental natural frequency. The results show that tibia has an anisotropic structure which has an important effect on bone parameters. Structural behaviour of the bone is described in terms of its anisotropic elasticity which is mostly depend on the complex stiffness. The real part of the complex elasticity determines bone system's acoustic energy storage capability and its imaginary part indicates its energy dissipation capability. The acoustic energy storage capability of the bone is found to be less than the energy dissipation capability of the bone.

Further work needs to be carried out to determine the strength of the bone from transfer function method using structural borne acoustic waves. More measurements should be undertaken on healthy and unhealthy male and female bone tibias to determine acoustical and mechanical parameters of them.

\section{Acknowledgement:}

This work has been supported by Research \& Enterprise / ID No: 1027 of Southampton Solent University, and by Acoustics Group of London South Bank University.

\section{References:}

1. Thomsen, J.S., Ebbesen, E.N. and Mosekilde, L. (2002) Age-Related Differences between Thinning of Horizontal and Vertical Trabeculae in Human Lumbar Bone as Assessed by a New Computerized Method. Bone, 31, 136-142.

2. Licata, A., 2009 Bone density vs bone quality: What's a clinician to do? Journal of Medicine. Volume 76 (6), pp.331-336

3. The world health report 2004: changing history. Geneva, World Health Organization, 2004.

4. Cooper C., Campion G., and Melton L. J., 3rd (1992) Hip fractures in the elderly: a world-wide projection. Osteoporosis International 2, pp.285-289.

5. Rachner, T.D., S. Khosla, and L.C. Hofbauer, 2011, Osteoporosis: now and the future. The Lancet. 377(9773): p. 1276-1287.

6. Kanis, J. and O. Johnell, 2005, Requirements for DXA for the management of osteoporosis in Europe. Osteoporosis International. 16(3): p. 229-238.

7. Cranney, A., et al., 2007, Low bone mineral density and fracture burden in postmenopausal women. Canadian Medical Association Journal. 177(6): p. 575-580.

8. Stone, K.L., et al., 2003, BMD at multiple sites and risk of fracture of multiple types: long-term results from the Study of Osteoporotic Fractures. Journal of Bone and Mineral Research. 18(11): p. 1947-1954.

9. Lang, T.F., 2010, Quantitative Computed Tomography. Radiologic Clinics of North America. 48(3): p. 589-600.

10. Miller, P.D., et al., 1999, Bone densitometry: the best way to detect osteoporosis and to monitor therapy. Journal of Clinical Endocrinology and Metabolism. 84(6): p. 18671871. 
11. Damilakis, J., et al., 2010, Radiation exposure in X-ray-based imaging techniques used in osteoporosis. European Radiology. 20(11): p. 2707-2714.

12. Rüegsegger, P., B. Koller, and R. Müller, 1996, A microtomographic system for the non-destructive evaluation of bone architecture. Calcified tissue international. 58(1): p. 24-29.

13. Langton, C.M., S.B. Palmer, and R.W. Porter, 1984, The measurement of broadband ultrasonic attenuation in cancellous bone. Engineering in medicine. 13(2): p. 89-91.

14. Marín, F., et al., 2006, Relationship Between Bone Quantitative Ultrasound and Fractures: A Meta-Analysis. Journal of bone and mineral research. 21(7): p. 11261135.

15. Trimpou, P., et al., 2010, High correlation between quantitative ultrasound and DXA during 7 years of follow-up. European journal of radiology. 73(2): p. 360-364.

16. Perre, G. V. D., and Lowet, G, 1996. In vivo assessment of bone mechanical properties by vibration and ultrasonic wave propagation analysis. Bone. Volume 18, No 1, 29S35S.

17. Perre, G. V. D., Lowet, G., Christenser, A. B., 1991. In vivo assessment of bone quality by vibration and wave propagation techniques part II. Proceedings of the COMC-BME II .2.6 Meeting, Durham, UK, April 11-13, apge 7-38.

18. Hobatho, M. C., Darmana, R., Barrau, J.J., Laroze, S., Morucci, J. P., 1988. Natural frequency analysis of human tibia. Biomechanics of bone and soft tissue. IEEE $10^{\text {th }}$ Annual international conference.

19. Bochud N., Vallet Q., Minonzio J. G., and Laugier P. 2017. Predicting bone strength with ultrasonic guided waves. Scientific reports. 7:43628. 1-14.

20. Attenborough K., Qin Q., Fagan M. J., Shin H-C., and Langton C. M., 2005. Measurements of tortuosity in stereolithographical bone replicas using audio-frequency pulses. Journal of the Acoustical Society of America 118, pp. 2779-2782.

21. Aygün H., Attenborough K., Lauriks W., and Langton M.C., 2010, Ultrasonic wave propagation in Stereolithographical bone replicas. Journal of the Acoustical Society of America 127 (6), pp. 3781-3789.

22. Aygün H., Attenborough K., Lauriks W., Rubini P.A., and Langton M.C., 2011, "Wave propagation in Stereolithographical (STL) bone replicas at oblique incidence.” Applied Acoustics 72 (7), pp. 458-463.

23. Aygün H., and Barlow C., 2015, Ultrasonic wave propagation through porous ceramics at different angles of propagation. Applied Acoustics 88, pp. 6-11.

24. Hughes E. R., Leighton T. G., White P. R., and Petley G. W., 2007 Investigation of an anisotropic tortuosity in a Biot model of ultrasonic propagation in cancellous bone. $J$. Acoust. Soc. Am. 121 568-574.

25. Lee K. I., and Choi M. J., 2007, Phase velocity and normalized broadband ultrasonic attenuation in Polyacetal cuboid bone-mimicking phantoms. J. Acoust. Soc. Am. 121 (6) EL263-EL269.

26. Sebaa N., Fellah Z., Fellah M., Ogam E., Wirgin A., Mitri F., Depollier C., and Lauriks W., 2006 Ultrasonic characterisation of human cancellous bone using the Biot theory: Inverse problem. J. Acoust. Soc. Am. 120 1816-1824. 
27. Lee K. I., and Yoon S. W., 2006 Comparison of acoustic characteristics predicted by Biot's theory and the modified Biot-Attenborough model in cancellous bone. $J$. Biomech. 39 364-368.

28. Fellah Z. E. A., Chapelon J. Y., Berger S., Lauriks W., and Depollier C. 2004 Ultrasonic wave propagation in human cancellous bone: Application of Biot theory. J. Acoust. Soc. Am. 116 61-73.

29. Lee K. I., Roh H.-S., and Yoon S. W., 2003 Acoustic wave propagation in bovine cancellous bone: Application of the Modified Biot-Attenborough model. J. Acoust. Soc. Am. $1142284-2293$.

30. Hughes E. R., Leighton T. G., Petley G. W., and White P. R., 1999 Ultrasonic propagation in cancellous bone: A new stratified model. Ultrasound Med. Biol. 25 811821.

31. Hosokawa A., and Otani T., 1997 Ultrasonic wave propagation in bovine cancellous bone. J. Acoust. Soc. Am.101 558-562.

32. McKelvie M. L., and Palmer S. B., 1991 The interaction of ultrasound with cancellous bone. Phys. Med. Biol. 36 1331-1340.

33. Biot M. A., 1956 Theory of propagation of elastic waves in a fluid saturated porous solid, I. Low frequency range. J. Acoust. Soc. Am. 28 168-1178.

34. Biot M. A., 1956 Theory of propagation of elastic waves in a fluid saturated porous solid, II. High frequency range. J. Acoust. Soc. Am. 28 179-191.

35. Prendergast, P., 1997, Finite element models in tissue mechanics and orthopaedic implant design. Clinical Biomechanics. 12(6): p. 343-366.

36. Langton, C.M., S. Pisharody, and J.H. Keyak, 2009, Comparison of 3D finite element analysis derived stiffness and BMD to determine the failure load of the excised proximal femur. Medical Engineering and Physics. 31(6): p. 668-672.

37. Buckley, J.M., K. Loo, and J. Motherway, Comparison of quantitative computed tomography-based measures in predicting vertebral compressive strength. Bone, 2007. 40(3): p. 767-774.

38. Keyak, J.H., et al., 1998, Prediction of femoral fracture load using automated finite element modelling. Journal of Biomechanics. 31(2): p. 125-133.

39. Langton et al., 2018, Estimation of mechanical stiffness by finite element analysis of ultrasound computed tomography (UCT-FEA); a comparison with X-ray microCT based FEA in cancellous bone replica models. Applied Acoustics. (Accepted for publications: APAC_2017_474)

40. Aygun, H., Barlow, C., Yule, L., and Liu, S. Y., 2015 Sound Propagation through bone tissue. Proceedings of the Institute of Acoustics. Vol. 37. Pt. 2, pp.234-238.

41. Rao, S. S., 2004. Mechanical Vibration. Fifth edition. Pearson.

42. Drake, R. L., Vogl, A. W., and Mitchell, A. W. M. 2010. Gray’s anatomy for students. $2^{\text {nd }}$ edition.

43. Beranek, L. L., and Ver I. L., 1992. Noise and vibration control engineering: Principles and Applications. First edition. Wiley Interscience. 